TRANSACTIONS OF THE

AMERICAN MATHEMATICAL SOCIETY

Volume 183, September 1973

\title{
FINITE GROUPS WITH NICELY SUPPLEMENTED SYLOW NORMALIZERS
}

\author{
BY
}

\section{DAVID PERIN}

\begin{abstract}
This paper considers finite groups $G$ whose Sylow normalizers are supplemented by groups $D$ having a cyclic Hall $2^{\prime}$-subgroup. $G$ is solvable and all odd order composition factors of $G$ are cyclic. If $S \in \mathrm{Syl}_{2}(D)$ is cyclic, dihedral, semidihedral, or generalized quaternion, then $G$ is almost supersolvable.
\end{abstract}

Let $D$ denote the class of finite groups $D$ which satisfy:

(*) $D=S T$, where $S \in \operatorname{Syl}_{2}(D)$ and $T$ is cyclic group of odd order.

We say $G$ is $\mathscr{D}$-supplemented if $G$ is finite and every Sylow normalizer in $G$ has a supplement $D \in \mathscr{D}$.

Theorem 1. D-supplemented groups are solvable.

Proof. Assume the theorem is false, and let $G$ be a counterexample of minimal order. Since any homomorphic image of $G$ is $D$-supplemented, $G / N$ is solvable for any $1 \neq N \triangleleft G$. Thus, $G$ has a unique minimal normal subgroup $M$. $M$ is nonsolvable, and so 2 divides $|M|$ by the Feit-Thompson Theorem. Choose $P \in$ $\operatorname{Syl}_{2}(M)$ and $Q \in \operatorname{Syl}_{2}(G), P \leq Q$. By the Frattini argument $G=M N(P)$. Let $D \in D$ be a supplement for $N(Q)$. Since $Q \in \operatorname{Syl}_{2}(G)$, we can assume $D$ is cyclic of odd order. Choose a subgroup $H \geq N(P)$ which is maximal in $G$. Since $N(P) \geq N(Q)$, $D$ is a supplement for $H$. $(D \cap H)^{G}=(D \cap H)^{H} \leq H$. If $D \cap H \neq 1$, then $\bar{M} \leq$ $(D \cap H)^{G} \leq H$, a contradiction. Consequently, $N(P)=N(Q)$ is maximal in $G$, and $D$ is a complement for $N(P)$. $G$ has a faithful primitive representation on the $d=|D|$ cosets of $N(P)$, and $D$ is regularly represented. If $d$ is not prime, then $D$ is a $B$-group $[8,25.2]$, and so $G$ is 2 -transitive. Otherwise $d$ is prime, and $G$ is 2-transitive by a theorem of Burnside.

Recent results of Shult and O'Nan classify 2-transitive groups $H$ in which $H_{\alpha}$ is a 2-local subgroup. If $T=O_{2}\left(H_{\alpha}\right)$ is semiregular on $\Omega-\{a\}$, then Shult's Fusion Theorem (see [5]) implies that $H$ has a regular normal subgroup, or $N \unlhd H \leq \operatorname{Aut}(N)$, where $N$ is isomorphic to $\operatorname{PSL}_{2}\left(2^{a}\right), \operatorname{PSU}_{3}\left(2^{a}\right)$, or $\operatorname{Sz}_{2}\left(2^{2 a+1}\right)$ in its standard 2-transitive permutation representation. (We need Shult's result only in the case $\mathrm{O}_{2}\left(G_{\alpha}\right) \in \operatorname{Syl}_{2}(G)$. This special case follows from Suzuki's work

Received by the editors July 28, 1972.

AMS (MOS) subject classifications (1970). Primary 20D10, $20 \mathrm{D} 40$. 
on finite groups with independent Sylow 2-subgroups [7].) If $T$ is not semiregular, then work of O'Nan [6] implies that $H$ has a regular normal subgroup or $N \unlhd H \leq$ Aut $(N)$, where $N \simeq \operatorname{PSL}_{n}\left(2^{a}\right)$. Since $G$ has no regular normal subgroup and $O_{2}\left(G_{a}\right)$ is a Sylow 2-subgroup of $G$, the only possibility is $N \unlhd G \leq$ Aut $(N)$, where $N \simeq \operatorname{PSL}_{2}\left(2^{a}\right), \operatorname{PSU}_{3}\left(2^{a}\right)$, or $\mathrm{Sz}_{z}\left(2^{2 a+1}\right)$. In these cases one easily finds a prime $p$ and $S \in \operatorname{Syl}_{p}(G)$ so that $N(S)$ has no supplement $D \in \mathcal{D}$. For example, if $G \simeq \operatorname{PSL}_{2}(4)$ take $p=3$, and if $G \simeq \mathrm{P \Gamma L}_{2}(4)$ take $p=2$.

Remark. If $G \simeq \operatorname{PSL}_{2}\left(2^{a}\right)$ and $S \in \operatorname{Syl}_{2}(G)$, then $N(S)$ has a cyclic complement of odd order.

Theorem 2. If $G$ is D-supplemented then every chief factor of $G$ of odd order is cyclic.

Proof. Let $G$ be a counterexample of minimal order. A result of Huppert [4, VI. 8.6] implies that $\Phi(G)=1$. $G$ has a unique minimal normal subgroup $M$. Since $G$ is solvable, $M$ is an elementary abelian $p$-group. $p$ is odd. Set $P=O_{p}(G)$. $P$ is elementary abelian since $\Phi(P) \leq \Phi(G)=1$.

There is a prime $q \neq p$ and a $q$-group $1 \neq Q<G$ so that $P Q \unlhd G$. $P=$ $[P, Q] \times C_{P}(Q)$. Since $[P, Q] \neq 1$ and $C_{P}(Q)$ and $[P, Q]$ are normal in $G$, $C_{P}(Q)=1$. $G$ is a split extension of $P$ by $N(Q)$. If $Q \leq Q_{1} \in \operatorname{Syl}_{q}(G)$, then $N(Q) \geq N\left(Q_{1}\right)$. Consequently, $N(Q)$ has a supplement $D \in \mathfrak{D}$. $D$ contains an element $\bar{x}$ of order $p^{m}=|P|$. The image $\bar{x}$ of $x$ in $\bar{G}=G / P$ has order at least $p^{m-1}$. Since $\bar{G}$ is isomorphic to a subgroup of $\mathrm{GL}_{m}(p), p m>p^{m-1}$. Hence, $m=2$. $G$ contains an element of order $p^{2}$, and so $p$ divides $|\bar{G}|$. But $O_{p}(\bar{G})=1$ and $\bar{G}$ is solvable. The only possibility is $p=3$ and $\bar{G} \simeq \mathrm{SL}_{2}(3)$ or $\mathrm{GL}_{2}(3)$. Then the normalizer of $S \in \operatorname{Syl}_{2}(G)$ has index 9 or 27 in $G$. However, $G$ contains no elements of order 9 , a final contradiction.

Let $\mathfrak{D}^{*}$ denote the class of finite groups $D$ which are the product of a cyclic group $T$ of odd order and a cyclic, dihedral, semidihedral, or generalized quaternion 2-group $S . T$ is a Hall $2^{\prime}$-subgroup of $D$ and $S \in \operatorname{Syl}_{2}(D) . D \in \mathfrak{D}^{*}$ implies $D \in \mathscr{D}$, so that $\mathfrak{D}^{*}$-supplemented groups are solvable. Buchthal [1] has show that certain solvable $\mathfrak{D}^{*}$-supplemented groups are either supersolvable or have $\Sigma_{4}$ as a homomorphic image.

Theorem 3. If $G$ is $\mathfrak{D}^{*}$-supplemented, then $G$ contains a normal subgroup $N$ such that every G-composition factor of $N$ is cyclic and $G / N$ is isomorphic to 1 , $A_{4}, \Sigma_{4}$, or one of the groups $\Gamma_{1}, \Gamma_{2}, \Gamma_{3}$ defined below.

The group $\Gamma_{1}$ is defined as follows. Let $W$ be an elementary abelian group of order 16. Choose $g \in$ Aut $(W)$ so that $|g|=3$ and $C_{W}(g)=1$. Let $S$ be a Sylow 2-subgroup of $N_{\text {Aur }(W)}(\langle g\rangle) \simeq \Gamma L_{2}(4) . S$ and $g$ generate a group $X$ of order 24 . 
Define $\Gamma_{1}$ to be the split extension of $W$ by $X$. The normalizer $N(R)$ of $R \in \operatorname{Syl}_{3}\left(\Gamma_{1}\right)$ has index 16 in $\Gamma_{1}$. The only supplements $D \in D^{*}$ for $N(R)$ are semidihedral or generalized quaternion groups of order 16. (These facts are established in the proof of Theorem 3.)

Suppose $W \simeq Z_{4} \times Z_{4}$. Let $a$ and $b$ be generators of $W$. Define automorphisms $g, x, z$, and $s$ of $W$ as follows.

1. $a^{g}=b^{-1}, b^{g}=a b^{-1}$,

2. $a^{z}=a^{-1}, b^{z}=b^{-1}$,

3. $a^{x}=a b^{2}, b^{x}=a^{2} b^{-1}$,

4. $a^{s}=b, b^{s}=a$.

The element $g \in$ Aut $(W)$ has order 3 , while $x, z$, and $s$ are involutions. $C_{\text {Aut }(W)}(g)=\langle g, x, z\rangle$ and $N_{\text {Aut }(W)}(\langle g\rangle)=\langle g, x, z, s\rangle=X . \Gamma_{2}$ is the split extension of $W$ by $X . S=\langle a, b, x, z, s\rangle$ is a Sylow 2-subgroup of $\Gamma_{2} . S$ contains no elements of order 16, and every element of order 8 in $S$ is conjugate to sa. $N_{S}(\langle s a\rangle)$ is a split extension of $\langle s a\rangle$ by the 4-group $\left\langle z b, a^{2}\right\rangle \cdot\langle s a, z b\rangle$ and $\left\langle s a, a^{2}\right\rangle$ are complements for $N_{S}(\langle g\rangle)$ in $S$, while $\left\langle s a, z b a^{2}\right\rangle \cap N_{S}(\langle g\rangle)=\langle s z\rangle$. Also, $\langle s a, z b\rangle$ is semidihedral, and $\left\langle s a, a^{2}\right\rangle$ is neither dihedral nor semidihedral. These facts yield the following result.

Lemma 1. $\Gamma_{2}$ is $\mathfrak{D}^{*}$-supplemented. Any proper subgroup of $\Gamma_{2}$ which contains $\langle a, b, g\rangle$ and is $\mathscr{D}^{*}$-supplemented is conjugate in $\Gamma_{2}$ to $\Gamma_{3}=\langle a, b, g, z, s\rangle$. Moreover, if $\Gamma=\Gamma_{2}$ or $\Gamma_{3}$ and $R \in \operatorname{Syl}_{3}(\Gamma)$, then $N(R)$ bas index 16 in $\Gamma$ and the only supplements $D \in \mathfrak{D}^{*}$ for $N(R)$ are semidibedral groups of order 16 .

Proof of Theorem 3. In the following discussion, $\Gamma$ denotes any one of the groups $\Gamma_{1}, \Gamma_{2}$, or $\Gamma_{3}$.

Let $G$ be a counterexample of minimal order. Choose $N \unlhd G$ of minimal order so that $G / N \simeq 1, A_{4}, \Sigma_{4}$, or $\Gamma$. (E.g., if $G$ has both $\Sigma_{4}$ and $\Gamma$ as homomorphic images, choose $N$ such that $G / N \simeq \Gamma$.) $N$ contains a unique minimal normal subgroup $M$ of $G$. $M$ is not cyclic. Theorem 2 implies that $M$ is a 2 group. Set $P=O_{2}(N) . C_{N}(P) \leq P$ and $O_{2}(G)=1$. Suppose $\Phi(P) \neq 1$. Then by induction each $G$-composition factor of $P / \Phi(P)$ is cyclic, and so $G / C_{G}(P / \Phi(P))$ is a 2 -group. Hence, $G / C_{G}(P)$ is a 2 -group $[3,5.1 .4]$, in which case $P \cap Z(G) \neq 1$. This contradiction implies that $P$ is elementary abelian.

Assume $P \neq N$. Then there is a prime $q \neq 2$ and a $q$-group $1 \neq Q<N$ so that $Q P$ is normal in $G . C_{P}(Q)=1$. Let $|P|=2^{m}$. If $C_{G}(P)=P$, then the proof of Theorem 2 shows that $2^{m-2}<2 m$, or $m \leq 5$. If $m<4$, there is no choice for $q$. If $m=5$, then $q=31$. But the normalizer in $\mathrm{GL}_{5}(2)$ of a group of order 31 has order 31 . 5. It follows that $P \in \mathrm{Syl}_{2}(G)$, and so $N(Q)$ is not $\mathfrak{D}^{*}$-supplemented. Thus, the only possibility is $m=4$ and $q=3$ or 5 . $N(Q)$ has a supplement $D$ 
which is cyclic, dihedral, semihedral, or generalized quaternion of order at least $|P|=16$. $D$ has no normal elementary abelian subgroup of order 4 , and so $|D \cap P| \leq 2$. Thus, $D P / P$ has order at least 8 . The normalizer in $\mathrm{GL}_{4}(2)$ of a cyclic group of order 5 is metacyclic group of order 60 . Consequently, $q=3$. Since $C_{P}(Q)=1$, the normalizer in $\mathrm{GL}_{4}(2)$ of $Q$ is $\Gamma_{2}(4)$. Since $G$ is solvable, the only possibility is that $N(Q)$ is a split extension of $Q$ by $D_{8}$ or $\Sigma_{4}$. In either case $O_{2}(G / P) \simeq Z_{2} \times Z_{2} \simeq C_{P}\left(O_{2}(G / P)\right)$. By induction $G / P$ acts reducibly on $P / C_{P}\left(O_{2}(G / P)\right)$, which is not the case. Therefore, $C_{G}(P)$ properly contains $P$, whence $N \neq G$. There is a group $P<K \unlhd G$ so that $K / P \simeq Z_{2} \times Z_{2} \cdot C_{P}(Q)=1$ and $[K, Q] \leq P$ imply $C_{K}(Q) \simeq Z_{2} \times Z_{2}[3,5.3 .15] . C_{K}(Q)=C_{K}(P Q)$ is normal in $G$. Then $K \leq C(P)$ implies $K=P \times C_{K}(Q)$, and so $K$ is elementary abelian. If $X \leq G$ let $\widetilde{X}$ denote the image of $X$ in $\widetilde{G}=G / C_{K}(Q)$. By induction $G$ has a normal subgroup $H \geq C_{K}(Q)$ so that $\widetilde{G} \widetilde{H} \simeq 1, A_{4}, \Sigma_{4}$ or $\Gamma$, and each $\widetilde{G}$ composition factor of $\widetilde{H}$ is cyclic. From the facts that $M$ is noncyclic, $M \cap C_{K}(Q)=1$, and $M$ is the only minimal normal subgroup of $G$ contained in $N$, it follows that $N$ is isomorphic to a subgroup of $\widetilde{G} / \widetilde{H}$. Thus, $Q \simeq Z_{3}$. Choose $S \in \operatorname{Syl}_{3}(G)$. Suppose $G / N \simeq \Gamma$. Then $G: N(S) \geq 64$. Consequently, there is a cyclic, dihedral, semidihedral, or generalized quaternion group $D$ of order at least 64 which is a supplement for $N(S)$. For $X \leq G$ let $\bar{X}$ denote the image of $X$ in $\bar{G}=G / N \simeq \Gamma$. Then $\bar{S} \in \operatorname{Syl}_{3}(\bar{G})$ and $N_{\bar{G}}(\bar{S})=\overline{N_{G}(S)}$. Thus, $\bar{D} \simeq D / D \cap N$ is a supplement for $N_{\bar{G}}(\bar{S})$. But $D \cap N \neq 1$ since $\Gamma$ has exponent 24 . Hence, $\bar{D}$ is cyclic or dihedral, whereas a $D^{*}$-supplement for $N_{\bar{G}}(\bar{S})$ in $\bar{G} \simeq \Gamma$ must be semidihedral or generalized quaternion of order 16. This contradiction implies $G / N \simeq A_{4}$ or $\Sigma_{4}$, whence $N \simeq A_{4}$, or $\Sigma_{4}$. Then $S \simeq Z_{3} \times Z_{3}, G: N(S)=16$, and $|G / K|$ divides 36. A Sylow 2-subgroup of $G / K$ is not cyclic of order 4 , and so the exponent of $G$ divides 12 . Hence, $N(S)$ does not have a supplement $D \in D^{*}$.

The only remaining case is $P=N$. Then $G \neq N$ and there is an element $g \in G$ of order 3. Assume $G / N \simeq \Gamma$. $C_{G}(N)$ does not contain $g$, for otherwise $G / C_{G}(N)$ is a 2 -group, and $N \cap Z(G) \neq 1$. Hence, $N: C_{N}(g) \geq 4$, and so $G: N(\langle g\rangle) \geq 64$. Since $G$ has exponent 24 or $48, N(\langle g\rangle)$ has no supplement $D \in D^{*}$. Thus, $G / N \simeq A_{4}$ or $\Sigma_{4}$. Set $K=O_{2}(G) . K / N \simeq Z_{2} \times Z_{2}$. Suppose $[N, K]=1$. Then $C_{N}(g)=1$, and by induction $|N|=4$. A supplement $D \in D^{*}$ for $N(\langle g\rangle)$ has order at least 16 , whence $|D \cap K| \geq 8$. Since $K$ has exponent 2 or $4, D \cap K$ is dihedral or quaternion. Thus, $K$ is a nonabelian group of order 16 and exponent 4. According to Burnside [2, p. 146] $\left|K^{\prime}\right|=2$, and so $N$ contains a subgroup of order 2 which is normal in $G$. This contradiction implies $[N, K]=U \neq 1$.

By induction each $G$-composition factor of $N / M$ is cyclic of order 2 . Consequently, $g$ centralizes $N / M$. Then $K=N[K, g]$ also centralizes $N / M$, so $U \leq M$. Thus, $U=M$ and $K$ centralizes $U$. Then $C_{U}(g)=1$. By induction 
$|U|=4$. Set $V=C_{N}(g)$, so that $N=V \times U$. Choose $H<K$ such that $H: N=2$. $C_{N}(H)$ contains $U$ and therefore is normalized by $g$. Then $C_{N}(H)=C_{N}\left(H^{8}\right)=$ $C_{N}\left(H H^{g}\right)=C_{N}(K)$. Since $C_{N}(K) \cap C_{N}(g)=1, C_{N}(H)=U$. Consequently, $|N|=8$ or 16 .

Suppose there is an element $x \in K$ so that the image $\bar{x}$ of $x$ in $\bar{K}=K / U$ has order 4. $x^{2} \in N$ since $K / N \simeq Z_{2} \times Z_{2}$, but $x \notin N$ since $N$ is elementary. Hence, $C_{K}\left(x^{2}\right)$ properly contains $N$, which is not the case. Consequently, $K / U$ is elementary abelian, and so $K / U=C_{K / U}(g) \times[K / U, g]$. Since $U=[U, g]<W=[K, g]$ and $C_{U}(g)=1, K$ is a split extension of $W$ by $V . W$ has order 16 and is normal in $G$. Since $W$ has exponent at most 4 and $\left|W^{\prime}\right| \neq 2, W$ is abelian.

Let $R=\langle g\rangle . G$ is a split extension of $W$ by $N(R) . N(R)$ acts faithfully on $W$. $N(R)$ has a supplement $D$ which is cyclic, dihedral, semidihedral, or generalized quaternion. Since $K$ has exponent 4 , the only possibility is $G / N \simeq \Sigma_{4}, D$ is a complement for $N(R)$, and $D$ is dihedral, semidihedral, or generalized quaternion of order 16. Suppose $W \simeq Z_{4} \times Z_{4}$. Then Lemma 1 yields $G \simeq \Gamma_{2}$ or $\Gamma_{3}$. This contradiction implies that $W$ is elementary abelian of order 16 , and $G$ is isomorphic to a subgroup of $\Gamma_{1}$. The nonidentity elements of $N \cup W$ have order 2, while all elements in $K-(N \cup W)$ have order 4. Since $D$ has no normal 4-group, $D \cap N=D \cap W \simeq Z_{2}$. Hence, $D \cap K$ is a quaternion group, and so $D$ is semidihedral or generalized quaternion of order 16. Moreover, $|D W|=|D||W| /|D \cap W|=$ $2^{7}$, so that $|G|=3 \cdot 2^{7}=\left|\Gamma_{1}\right|$. Then $G \simeq \Gamma_{1}$, a final contradiction.

Thus, $G$ has a normal subgroup $N$ so that every $G$-composition factor of $N$ is cyclic and $G / N \simeq 1, A_{4}, \Sigma_{4}, \Gamma_{1}, \Gamma_{2}$, or $\Gamma_{3} . N$ is the join of all groups $H \unlhd G$ which are supersolvably embedded in $G$, and so $N$ is unique.

Acknowledgement. The author would like to thank Professor W. R. Scott for several helpful conversations during the preparation of this article.

\section{REFERENCES}

1. D. Buchthal, On factorized groups, Trans. Amer. Math Soc. 183 (1973), 425-432.

2. W. Burnside, Theory of groups, 2nd ed., Dover, New York, 1955. MR 16, 1086.

3. D. Gorenstein, Finite groups, Harper and Row, New York, 1968. MR 38 \#229.

4. B. Huppert, Endliche Gruppen I, Die Grundlehren der math. Wissenschaften, Band 134, Springer-Verlag, Berlin and New York, 1967. MR 37 \#302.

5. W. Kantor and G. Seitz, Some results on 2-transitive groups, Invent. Math. 13 (1971), 125-142.

6. M. O'Nan, Illinois J. Math. (to appear).

7. M. Suzuki, Finite groups of even order in which Sylow 2-groups are independent, Ann. of Math. (2) 80 (1964), 58-77. MR $29 \# 145$.

8. H. Wielandt, Finite permutation groups, Lectures, University of Tübingen, 1954/55; English transl., Academic Press, New York, 1964. MR 32 \#1252.

DEPARTMENT OF MATHEMATICS, VIRGINIA POLYTECHNIC INSTITUTE AND STATE UNIVERSITY, BLACKSBURG, VIRGINIA 24061 